EUROPEAN LABORATORY FOR PARTICLE PHYSICS

CERN-PPE/97-72

3 July 1997

\title{
PROGRESS WITH DIAMOND OVER-COATED MICROSTRIP GAS CHAMBERS
}

B. Boimska\#, R. Bouclier, M. Capeáns, W. Dominik\# ${ }^{\#}$, G. Million, L. Ropelewski, F. Sauli, A. Sharma*', T. Temmel-Ropelewski

\begin{abstract}
We describe recent observations and measurements with Micro-Strip Gas Chambers coated, after manufacturing, with a thin diamond-like layer in order to increase their rate capability. Compared to the more widely used solution consisting in coating the insulating support with a conductive layer before photo-lithography (the so-called undercoating), over-coating has the advantage of avoiding possible problems with adherence of metals to the layer, damages during the etching process and reduced quality of the artwork resulting from imperfections or dust inclusions in the layer. Early tests have however indicated that, possibly because of damages to the layer due to electron and ion bombardment during the avalanche process, irreversible structural modifications and fatal breakdown could be encountered at very high integral radiation fluxes. The present paper summarizes these results, and describes recent developments demonstrating that a better choice of the parameters of the overcoat may allow to withstand the radiation doses anticipated for LHC detectors with the intrinsically simpler over-coating solution. We discuss also several possible applications of the use of thin, controlled resistivity layers for other families of detectors used or in development for CERN's high luminosity collider.
\end{abstract}

Submitted to Nuclear Instruments and Methods in Physics Research

\footnotetext{
\# On leave of absence from Institute of Experimental Physics, Warsaw University, Warsaw

* GRPHE, Université de Haute Alsace, Mulhouse, France
} 


\section{INTRODUCTION}

Micro-Strip Gas Chambers (MSGCs), introduced several years ago, are very promising position-sensitive detectors capable in principle of operating efficiently at particle rates exceeding $10^{6} \mathrm{~mm}^{-2} \mathrm{~s}^{-1}$. Aside from possible applications in many applied domains, MSGCs satisfy the requirements of tracking detectors planned for use at high luminosity colliders; large arrays of such devices are in construction for experiments at HERA B and LHC. Obtained with a variety of photo-lithographic methods, MSGCs in their simpler design consist of a set of parallel, thin metal strips, alternatively connected as anodes and cathodes, on an insulating support (the substrate). Overlaid by a gas volume delimited by a drift electrode, and with suitable potentials applied on electrodes, MSGCs allow to obtain a proportional gain of a few thousand or more, thus permitting detection and localization of ionizing radiation.

The intrinsic very high rate capability of the devices is a direct consequence of the fine granularity of the sensitive elements (typically strips are laid at a $100 \mu \mathrm{m}$ pitch) and of the small distance between anode and cathode strips, reducing considerably, to a few $\mu \mathrm{s}$, the collection time for the majority of the positive ions produced in the avalanches. Space charge induced field distortions, a limiting factor in conventional wire chambers, are therefore encountered only at extremely high rates.

Early in the development of the devices it was realized however that the nature of the substrate played a fundamental role in the general operating characteristics and in the rate capability. Domain polarization effects in the dielectric introduce disturbingly long time constants in the stabilization of gain after application of voltage and, most importantly, surface charging up due to temporary or permanent attachment of charges on the substrate seriously affect the detector, resulting in ratedependent gain modifications (usually a decrease). A large effort has been undertaken by many groups in order to study the above mentioned effects, and try to solve them by a suitable choice of the substrate; this work is summarized for example in the status reports of the RD-28 collaboration, a dedicated research project on MSGCs [1]. The use of bulk electron conducting glass, and various types of surface conditioning of insulating supports such as ion implantation, evaporation and sputtering of thin layers of controlled resistivity materials, were proposed. These studies have converged into suggesting that, as far as MSGCs are manufactured on a substrate having surface resistivities between $10^{13}$ and $10^{16} \Omega / \square$ and electronic conductivity, both polarization and surface charging up processes can be eliminated. Another process negatively affecting the lifetime of the detectors exposed to sustained irradiation, namely ageing, is substantially reduced when devices are built on supports having moderate resistivity.

The first attempts to use Diamond-Like Carbon (DLC) thin film deposition technique have been described some time ago [1]; the samples obtained, however, did not have either the required resistivity, or sufficiently good surface quality for MSGC operation. Recently, a method for obtaining the desired surface resistivity using a thin DLC layer has been developed [2], employing Plasma Assisted Chemical Vapour Deposition (PACVD), that consists in creating the conditions of a plasma discharge in a low-pressure molecular gas containing carbon $\left(\mathrm{CO}_{2}, \mathrm{CH}_{4}, \ldots\right)$. The thin film is then grown rather uniformly, with a morphology that depends on the various reaction parameters. While obtaining the desired value of resistivity implies the use of a proprietary surface treatment, the deposition of diamond-like layers with major properties, hardness and thermal conductivity close to that of natural diamond, is by itself a well known industrial process, and allows to obtain large, uniform coatings on a variety of supports at moderate costs over extended areas.

Very high rate capability, stable operating conditions and absence of ageing processes up to very high integral doses have been demonstrated [3]; the diamond- 
like film, typically $100 \mathrm{~nm}$ thick, is applied on the bare substrate (thin boro-silicate glass) before photo-lithography.

Many attempts have been made to invert the conditioning order, depositing the controlled resistivity layer on the already manufactured MSGC [4,5], an intrinsically simpler process since it avoids possible problems with the adherence of the metals used for photo-lithography on the layer; also, over-coating is more tolerant to the presence of defects or residual dust in the coating that can affect the quality of the etching. Promising results showing good short-term operation characteristics of DLC over-coated MSGC on boro-silicate glass supports have been already reported [3]. In the over-coating solution however, charges produced in the avalanche (electrons and ions) have to be neutralized by electric currents across the semi-conducting layer; this opens up the possibility of damage to the layer due to thermal and chemical effects. The long-term survivability of such an approach remains therefore to be demonstrated, particularly for the use of the detector at very high instant and integral rates.

The present paper describes our experience on medium- and long-term operation of over-coated MSGCs.

\section{EXPERIMENTAL SET-UP AND TEST CHAMBER CONSTRUCTION}

For the measurements described here we have used several chambers with identical electrode patterns realized by wet etching in chromium ${ }^{1}$ on $10 \mathrm{~cm}^{*} 10 \mathrm{~cm}$ boro-silicate D- $263^{2}$ glass plates $300 \mu \mathrm{m}$ thick. After etching, the plates were coated with a CVD process using proprietary technology ${ }^{3}$ by a thin diamond-like film in a range of thickness and surface resistivities. All MSGCs used in the present study have strip widths of $100 \mu \mathrm{m}$ for cathodes and $7 \mu \mathrm{m}$ for anodes, with a cathode-anode pitch $100 \mu \mathrm{m}$ (distance center-to-center). Detectors were tested in argon - dimethylether (DME) gas mixture in equal proportions in nominally clean conditions (only vacuumgrade materials like stainless steel, aluminium, glass and ceramics were used for the detector box construction and gas filling system [6]). The following structures have been tested:

- MSGC \#1: $1500 \AA$ thick film over-coat with surface resistivity $1.1 * 10^{15} \Omega / \square$ (measured at $590 \mathrm{~V}$ between anode and cathode) with a continuous electrode on the opposite side of the plate (back-plane electrode); read-out from cathodes (cathodes grounded, positive polarity voltage applied to anodes). Back-plane electrode grounded.

- MSGC \#2: $500 \AA$ thick film of $2 * 10^{14} \Omega / \square$ (measured at $570 \mathrm{~V}$ test voltage) with back-plane electrode; read-out from anodes (anodes grounded, negative polarity voltage applied to cathodes); back-plane electrode grounded.

- Several chambers of high surface resistivity without back-plane electrode: $500 \AA$ thick film structures of resistivity varying between $10^{15} \Omega / \square$ and few times $10^{16}$ $\Omega / \square$; read-out from anodes (anodes grounded, negative polarity voltage applied to cathodes). These detectors were obtained by surface resistivity tuning of the MSGC \#2 using the temperature conditioning technique described later in this paper.

The detector structure with a stainless-steel grid at $7 \mathrm{~mm}$ distance from the strip plane has been enclosed in a gas tight stainless-steel box with $100 \mu \mathrm{m}$ aluminium window of the size corresponding to the dimensions of the detector [7]. A negative voltage was applied to the grid in order to provide a drift and charge-collection field; in most of measurements reported here a relatively low drift field $(2.2 \mathrm{kV} / \mathrm{cm})$ has

\footnotetext{
${ }^{1}$ Baumer IMT Masken und Teilungen AG, Greinfensee, Switzerland

${ }^{2}$ DESAG - Deutsche Spezialglass AG, Grüneplan, Germany

${ }^{3}$ SURMET Corp., Burlington MA, USA
} 
been used. For this value of field, $25 \%$ of ions from avalanches are collected by the drift electrode.

Powered strips were connected together and to the high voltage source (positive or negative, depending on the read-out scheme) through a protection resistor; read-out groups, consisting of 20 strips each and kept on ground potential, were individually accessible. For high rate irradiation, an X-ray generator delivering the iron fluorescence line of controlled intensity has been employed.

In the measurement of long term stability of the detector the double beam method, recently introduced in order to overcome some limitation of correction procedures [6], has been used. Together with the main irradiated spot, a second region in another group of strips with separate read-out in the MSGC was simultaneously exposed to an attenuated fraction of the X-ray beam; the ratio of intensities in the high and low irradiation regions was about one order of magnitude. Assuming that ageing would not occur in the region exposed to the low dose, the ratio of pulse height measured on the two spots provides a correction-free information on changes in gain due to intense irradiation. This procedure permits to correct medium-term pulseheight fluctuations caused by external parameters (temperature, pressure, humidity, gas mixture stability, etc.). A single-wire counter installed in the same gas line and exposed to the attenuated X-ray beam from the same source has been operated permanently during the measurement period providing complementary information about the fluctuations of external parameters; monitoring of the pulse height from this counter permits to correct on the fluctuations of the MSGC's electronics. During the tests, both detectors were continuously irradiated and current, pulse height and counting rate were recorded. The detailed description of the experimental set-up and the correction procedures can be found in [6]. The MSGCs were illuminated with an effective beam area (on the detector surface) between 3 and $5.5 \mathrm{~mm}^{2}$, and a detected flux up to $10^{6} \mathrm{~s}^{-1} \mathrm{~mm}^{-2}$ could be obtained.

A possible source of long-term stability variations, related to the size of the beam spot with respect to the width of the active chamber area, should be mentioned here. Apart from the group of strips selected for irradiation, the rest of strips were not connected (floating) during our tests. In this situation the charge gain on the edge strips of the irradiated group is higher than the gain on central ones, and may approach the limit of operation; indeed, under high flux irradiation the edges of the irradiated section are exposed to more severe conditions. This may provoke local damages initiating faster degradation of the detector performance with time, thus, biasing the measurement of detector ageing (apparent shorter life).

\section{EXPERIMENTAL MEASUREMENTS AND RESULTS}

\section{MSGCs:}

Three types of effects have been observed during extensive operation of

- Gain slewing: short-, medium- and long-term changes in detected pulse height caused by surface charging up, ions migration in the MSGC support, support polarization under electrostatic field and other temporary phenomena. The effect of these processes on the pulse height is fully reversible; the parameters of the chamber reset to their initial values, albeit often with long recovery time-constant, after removal of the cause.

- Ageing: irreversible degradation of chamber parameters (pulse height, energy resolution, rate capability). It may be caused by polymers or pollutants deposition on the detector surface under avalanches, or be related to permanent modification of the surface resistivity.

- Life-time (lethal dose): time of operation, or total collected charge above which the detector structure gets severely and permanently damaged. Beyond this limit the chamber does not perform properly even at low counting rates in the damaged area; the rest of the surface however may be still unaffected and fully operational. 
Surface and strips may be locally damaged by reactive species from the gas, by charges from the avalanches, by ions from the support or by micro-discharges induced by defects appearing on the charge amplifying structure.

Precise measurements of the long-term behaviour of pulse-height and of the recovery time allow to identify the type of dominant effect, and to make an estimate of the detector performance in given experimental conditions.

\subsection{Detector operation characteristics}

Over-coated MSGCs, with surface resistivity in the range $10^{14}$ to $10^{16} \Omega / \square$, are easy to operate; they reach nominal gain conditions immediately after first application of the voltage. No conditioning at lower voltages is needed.

The charge gain shows an exponential from the anode-cathode voltage difference (Fig. 1). One can reach a charge gain above 5000 without discharges in argon-DME (50-50) mixture for $6 \mathrm{keV} \mathrm{X}$-ray at moderate radiation rates. The precise quantitative correlation between charge gain and surface resistivity are difficult to establish for the large size detector, because the resistivity measured is the average value over whole length of a group of strips and during the charge gain measurement only a small fraction of the surface is irradiated.

The results confirm that for a given applied voltage the charge gain is higher for the chamber with higher surface resistivity. This effect is correlated with the shape of the electric field in the vicinity of the anode strips [8]; the field is stronger in the anode region for higher surface resistivity, and field lines are more concentrated on anode strips. The current between anodes and cathodes makes the charge distribution on the surface more uniform thus diffusing effective amplifying field.

The maximum charge gain (the gain at the limit of accidental discharges at the counting rate of about $10^{3} \mathrm{~Hz} / \mathrm{mm}^{2}$ ) seems also to be correlated with the surface resistivity; for the chambers of $10^{16} \Omega / \square$, the maximum gain is $2-3$ times higher than it is in the case of the resistivity in the range $10^{14}-10^{15} \Omega / D$. This has only indicative character; the maximum gain is obviously limited by the quality of the artwork and deposits on the surface. These factors, controllable only to a limited extent, may bias the result.

The leakage current as a function of applied voltage shows small deviation from Ohm's law (Fig. 2). This behaviour is expected: thin diamond-like carbon layers exhibit some properties of semiconductive materials, and their conductivity is temperature sensitive. With larger power dissipation on the resistive surface, the temperature of a thin resistive layer rises slightly and we observe a small decrease of surface resistivity. The shift in resistivity is very small in the range of working voltage of the MSGC and, at high surface resistivity, it has no noticeable consequence for the detector operation.

\subsection{Long term stability of operation.}

During long-term exposures, pulse heights from high and low irradiated MSGC areas, as well from the single wire counter were monitored every 15 minutes at momentarily lowered radiation flux in the MSGC. The avalanche current on the high voltage strips and on the drift electrode were measured at the high radiation flux. All MSGCs were tested at a nominal charge gain of 1500, measured at the counting rate of $10^{3} \mathrm{~Hz} / \mathrm{mm}^{2}$.

The raw data of the 200 hours test run of the MSGC\#2 are presented in the figure 3. Fluctuations of measured amplitudes are clearly correlated, and related to ambient temperature and atmospheric pressure variations. During the measurement, the high irradiated area of the chamber has been exposed to a very high radiation flux 
resulting in large local current of about $7 \mathrm{nA} / \mathrm{mm}^{2}\left(50 \mu \mathrm{C}\right.$ hour $\left.{ }^{-1} \mathrm{~cm}^{-1}\right)$ and at the end of the test run the flux has been increased $\left(10 \mathrm{nA} / \mathrm{mm}^{2}\right)$.

After a certain amount of integrated charge from avalanches, sudden discharges appeared in the highly irradiated area locally degrading the MSGC surface. The chamber could hold nominal voltages, but even a moderate radiation flux in the damaged area provoked discharges. On optical inspection after opening the detector, one could easily recognize the highly irradiated damaged area: rough surface with melted edges of both cathode and anode strips. The zone of damages was limited to the region of intense irradiation. This final degradation of the surface has been definitely set by discharges in the gas in the irradiated region; the state of the surface before local violent discharges needs further investigations.

The long-term behaviour of over-coated MSGC appears clearly after normalization of the pulse height in the highly irradiated area (ph1) using the monitor pulse (ph2) from the low irradiated regions (Fig. 4). The amplitude of the pk1 pulse at the beginning of the measurement has been normalized to the unity. The charge gain decreases by about $10 \%$ during the first irradiation period corresponding to the integrated charge of about $2 \mathrm{mC} / \mathrm{cm}$, and remains stable afterwards until the chamber reaches its life limit. Then, either abrupt degradation or continuous decrease of charge gain with energy resolution worsening is observed.

The very high avalanche current density (the expected local current density in CMS/LHC tracking detector is 20 times smaller) might cause the abrupt degradation of the weakened surface, appearing at an integrated charge of $12 \mathrm{mC} / \mathrm{cm}$. In separate measurement, using the same chamber irradiated in another location with 5 times lower X-ray flux, the degradation process begins at the same level of integrated charge but shows up as a gradual degradation of the charge gain; this can be interpreted as a typical ageing caused by organic deposit.

Two different chambers (MSGC\#1 and MSGC\#2), having different thickness and resistivities of the diamond coating, reached the operation limit at about 12 $\mathrm{mC} / \mathrm{cm}$ of strip length with different behaviour above this limit. One can conclude that, in first approximation, the life limit of over-coated MSGCs on D-263 glass having a back-plane electrode with DLC coating of thickness between $500 \AA$ and $1500 \AA$ and resistivity between $10^{14}$ and $10^{15} \Omega / \square$ is similar and around $10 \mathrm{mC} / \mathrm{cm}$.

Measurement of gain of the chamber at nominal voltages corresponding to the charge gain of 1500 , at low radiation rate, confirms the local character of the degradation in the detector performance (Fig. 5); the charge gain drops in the damaged area only. The width of this area corresponds to the width of the irradiation spot. The rest of the surface is fully operational and allows very good stability of operation; energy resolution in the damaged area is degraded. The local character of damage facilitates repetitive studies on the same detector.

Very interesting is the initial drop of the absolute gain, systematically observed for diamond over-coated MSGCs on ion-conducting glass supports. The origin of the effect is not yet well understood, but some features have been experimentally established. The decrease of relative gain is a function of integrated charge and it is independent of the current density (Fig. 6); it exhibits an exponential character with a decay constant of about $1 \mathrm{mC} / \mathrm{cm}$.

The process of initial gain drop is reversible; the gain of the detector slowly recovers after removal of the radiation source (Fig. 7). This temporary gain slewing is not caused by ageing; it may be attributed to slow ion migration in the support under the influence of avalanche currents. The effect of the dynamic polarization of the substrate should be less pronounced at higher surface conductivity, when the dark current on the surface screens the electrostatic induction from the bulk.

Measurement of the gain recovery process permits to evaluate a recovery time constant of about 80 hours, exponential in time. Knowledge of the decay constant allows to make predictions of the chamber operation stability in given experimental conditions; if the integrated current in single experimental run is a small fraction of 1 
$\mathrm{mC} / \mathrm{cm}$ of strip and runs are separated by long periods when the detector is off, the gain slewing will be negligible.

Measurements of rate capability (Fig. 8) show constant charge gain of the detector with surface resistivity of $2.4 * 10^{15} \Omega / \square$ up to maximum radiation intensity of $2^{*} 10^{6} \mathrm{~Hz} / \mathrm{mm}^{2}$. An increase of charge gain with rate, for rates above $10^{4} \mathrm{~Hz} / \mathrm{mm}^{2}$, is observed for the detector with surface resistivity of $2.2 * 10^{16} \Omega / \square$. The increase of gain with the irradiation rate is fully reversible and shows no hysteresis effect. At this level of the charge density, an uncoated MSGC on D-263 glass support exhibits usually a gain decrease with rate $[2,4,9]$. The gain increase, with the avalanche rate, of the coated MSGC with very high surface resistivity may lead to the lowering of the discharge limit of the detector. One can conclude that a resistivity of the coating in around of $10^{15} \Omega / \square$ is appropriate from the point of view of rate capability and maximum charge gain limit.

A study of long-term behaviour of the high surface resistivity MSGC shows good operation stability up to accumulated charge on strips of $50 \mathrm{mC} / \mathrm{cm}$ (Fig. 9). The initial drop of gain is clearly visible. The maximum integrated charge per unit length of strip is almost 5 times higher than for the lower resistivity chamber, before reaching abrupt discharges. During this measurement the drift field strength was 470 $\mathrm{V} / \mathrm{mm}$ (more than twice higher than in previous measurements); consequently, the current flowing to the MSGC cathodes was 2 times lower, this would affect ageing rate. The results of systematic measurements of the current sharing as a function of the drift field is published separately [10].

In our measurement, the chambers with higher surface resistivity show longer life-time. However, this may be attributed to different technique of the chamber preparation that make use of high temperature conditioning (see 3.4). One can expect that due to this procedure the surface was cleaner (residuals of fabrication process and other deposits are removed by heating at high temperature). The authors of Ref. [11] report the beneficial effect, on the long term operation stability, of extended vacuum bake of the detector made on glass.

\subsection{Radiation hardness of the diamond-like layer on D-263 glass.}

The effect of direct irradiation of the MSGC surface was studied, exposing MSGC \#2 to a high flux of X-rays of energy up to $20 \mathrm{keV}$ for a period of one week; the beam intensity was set to a level 10 times higher than the irradiation rate during the long-term stability tests. An estimated total dose of $40 \mathrm{kGy} / \mathrm{mm}^{2}$ was accumulated in the coated D-263 glass. During the exposure the chamber was filled with the standard operating gas, but voltages were not applied to the detector. Twice per day the chamber gain was measured for a short time at low radiation rate. As shown in Fig. 10, despite the intense irradiation, the detector does not show any gain change. This measurement confirms that gain slewing is due to the high density avalanche current only. One can observe, however, an influence of radiation (even for $6 \mathrm{keV} \mathrm{X}$ rays) on the D-263 substrate: the glass in the irradiated area becomes darker, however, no significant modification of the coated surface resistivity has been observed. There is also an effect of annealing: the darkening of the irradiated area gets less pronounced after certain time; increasing the temperature will speed up the recovery.

Measurements done by Pallarès at al [12] show that DESAG D-263 glass is very sensitive to radiation when exposed to a ${ }^{60} \mathrm{Co}$ source. The authors report the creation of a significant density of defects after few kGy, and correlate the number of defects with the level of glass darkening. It seems that the same type of glass modification is caused by low energy X-ray irradiation also. The effect of substrate modification due to radiation on the chamber operation is not evident, but it merits very careful study. 


\subsection{Study of thermal behaviour of amorphous carbon film.}

A systematic study of temperature effects on the characteristics of the coating was undertaken. Heating effects are an important concern of the manufacturing procedure; some stages of detector construction may require treatment at elevated temperatures.

The plates of D-263 glass were coated with a DLC films by different manufacturers. Samples \#1 and \#2 were coated with $2000 \AA$ DLC films ${ }^{4}$ and sample \#3 with $1000 \AA$ thick film ${ }^{5}$. Very different initial resistivities result from specific deposition conditions. The samples were heated in nitrogen atmosphere for two hours in progressively increasing temperature. After each run the plates were cooled, in nitrogen, and the resistivity of the coating was measured at room temperature. The results are shown in Figure 11. Data at $60{ }^{\circ} \mathrm{C}$ (temperature of CVD deposition) correspond to the initial resistivities. Up to $250{ }^{\circ} \mathrm{C}$ the resistivity of ICMC samples increases with the same slope; at $250{ }^{\circ} \mathrm{C}$ the resistivity is about 10 times higher than the initial one. At $320{ }^{\circ} \mathrm{C}$ we observed an increase of a conductivity with respect to the one at $250{ }^{\circ} \mathrm{C}$. The resistivity (measured only up to $200{ }^{\circ} \mathrm{C}$ ) of the sample from SURMET increases with slightly steeper slope.

After the thermal treatment in this range of temperature the characteristics of the coating remain stable, without noticeable degradation of mechanical properties. Hence, this procedure may be employed to tune the resistivity of a DLC layer to higher values. The increase of the resistivity is a common feature of DLC films, however the slope depends on the technology of deposition.

\section{DISCUSSION OF RESULTS AND CONCLUSIONS}

The over-coated Microstrip Gas Chambers operate well up to charge gain above 5000 for argon+DME (50:50) gas mixture.

Surface charging up process is not limiting the detector operation for coating resistivity about up to few times $10^{15} \Omega / \square$; detectors with the surface resistivity at the level of $10^{15} \Omega / \square$ exhibit stable and constant charge gain for radiation fluxes above $10^{6} \mathrm{~Hz} / \mathrm{mm}^{2}$. Study of the long-term behaviour confirms that the dominant observed effect in the normalized pulse height shift is slewing of the measured charge gain. The shift is of the order of $10 \%$ during the initial irradiation period, corresponding to about $2 \mathrm{mC} / \mathrm{cm}$ of integrated avalanche charge under exposure to the high intensity Xray flux of $6 \mathrm{keV}$, at the charge gain of 1500 . This is comparable to pulse-height variations due to pressure and temperature changes during a long operation period.

Measured detector's life-time (in units of detected charge) of $50 \mathrm{mC} / \mathrm{cm}$ of strip is close to the required level for tracking detectors at high luminosity.

The effect of pulse-height slewing does not change the detector operation in an irreversible way and therefore should not be called ageing which could be misleading. However, it may be the indication of processes occurring in the DLC layer thus limiting the life-time of the detector. The correlation between the slewing and the ageing is not clear. Understanding of the slewing will possibly allow to design over-coated MSGC structure with extended life-time in the high rate avalanche conditions.

A thickness of about $1000 \AA$ of the DLC coating seems to be adequate for over-coated MSGC. At this thickness, uniformity of the thin film parameters should be relatively easy to obtain in mass production. There is no clear evidence that the thickness of the coating film plays a role in short- and long-term operation of the detector, if the layer is thicker than few hundred angstroms. Adhesion of the thin "diamond" film to the chromium strips is very good. The surface sustains accidental

\footnotetext{
${ }^{4}$ deposited by ICMC Innovation Couches Minces, France

${ }^{5}$ deposited by SURMET Corp., Burlington MA, USA
} 
discharges in the gas without visible degradation. The metallic strips are well protected against single violent discharges by the thin DLC film. There was no effect of creation of short circuits between electrodes, provoked by discharges in the gas.

During the measurements gas purity and level of pollutants in the filling mixture were not monitored. It was found also, after finishing the measurements, that the cleaning procedure of the MSGC plates could lead to deposits of an organic liquid undetectable by eye. This could adversely affect detector operation by shortening its life under high rate of avalanches. One of the sources of limitation of the detector life may come from ions from the support bulk migrating to the "diamond" layer due to electric field, changing its mechanical or electrical properties. MSGCs supports made of materials that do not contain alkali ions may increase the life-time of the detector.

The resistivity of the DLC layer may be tuned to higher values by temperature conditioning of the coated plate. For the samples tested, controlled increase of the surface resistivity by more than one order of magnitude has been obtained. This process gives very reliable results for conditioning temperatures up to $250{ }^{\circ} \mathrm{C}$; the heat-treated film has stable properties and the mechanical quality remains good. The resulting resistivity is a function of the conditioning temperature only, and does not seem to depend on the duration of the treatment within the range of several hours. This makes the process easy to control.

The result obtained with the DLC layer exposed to the high rate of avalanches encourages one to imagine the extension of this type of electrode coating technique to other types of gaseous detectors.

One of the applications where this very stable coating could be of high value is the Resistive Plate Chamber [13] detector for the muon trigger in the Large Hadron Collider detectors. Coating the inner surface of the plates, constituting the detection gap, by a smooth layer of required resistivity should result in stable and well controlled detector operation. The DLC makes the surface very hard mechanically, which makes further assembling easier. The film is very stable chemically. The deposition of the thin film by PA CVD technique is done at temperatures below 50 ${ }^{\circ} \mathrm{C}$, so a large variety of materials can be coated.

The DLC coating may find application in the standard Multiwire Proportional Chambers. Cathodes with moderate resistivity may be made of thin films deposited on dielectric supports. Very smooth electrodes should allow to improve the discharge limit. Accidental discharges will be less violent because of significant resistivity of cathodes. Cathodes with the surface resistivity above $100 \mathrm{k} \Omega / \bar{\square}$ are transparent to the electro-magnetic wave induced by a charge avalanche in the gas [14]; this allows to install read-out electrodes outside the sensitive volume of the detector. Thus, detector construction becomes easier and one gains more freedom for shaping of the read-out electrode. Moreover, placing the read-out electrode at a larger distance from the anode wire plane has an additional advantage: one can decrease the number of readout channels preserving the resolution. This would allow the significant cost saving in a large scale detecting systems. Cathode Strip Chambers (CSC) for muon tracking system of the Compact Muon Solenoid [15] detector may reduce cost by choosing this solution. 


\section{REFERENCES}

[1] F. Sauli, RD-28 Status Reports CERN/DRDC/93-34 (1993) and CERN/DRDC/9445 (1994).

[2] R Bouclier et al, High rate operation of micro-strip gas chambers on diamondcoated glass. Nucl. Instr. Methods A369 (1996) 328.

[3] R. Bouclier et al, High rate operation of Micro-Strip Gas Chambers. IEEE Trans. Nucl. Sci. NS-43(3) (1996) 1220.

[4] R. Bouclier et al, MSGC with thin electron-conducting layers. Proc. Int. Workshop on Micro-Strip Gas Chambers, Legnaro Oct. 13-14, 1994, p. 39.

[5] M. Salomon at al, IEEE Trans. Nucl. Sci. NS-41 (1994) 817.

[6] R. Bouclier et al, Ageing of microstrip gas chambers: problems and solutions., CERN-PPE/96-33, 25 January 1996.

[7] L. Shekhtman, Study of gain limits in MSGC. CMS TN 95-045, May, 1995.

[8] J.J. Florent et al, The electrostatic field in microstrip chambers and its influence on detector performance. Nucl. Instr. Methods A329 (1993) 125.

[9] R. Bouclier et al, Development of micro-strip gas chambers for high rate operation. Nucl. Instr. Methods A367 (1995) 168.

[10] A. Barr et al, Diamond over-coated Microstrip Gas Chambers for high rate operation. presented at $5^{\text {th }}$ International Conference on Advanced Technology and Particle Physics, Como, 7-11 October, 1996.

[11] B.D. Ramsey et al, A Large-Area Microstrip-Gas-Counter for X-Ray Astronomy. NASA Space Science Laboratory preprint, to appear in Nucl. Instr. Methods.

[12] A. Pallarès et al, Substrate effects on Micro-strip Gas Chamber behaviour under irradiation. CRN 96-13 (1996), International Workshop on Microstrip Gas Chambers, Lyon, 1995, p.221.

[13] G. Wrochna (CMS Collaboration), RPC based muon trigger for the CMS detector at LHC. Proceedings of the Conference on Resistive Plate Chambers in Particle Physics and Astrophysics, Pavia 1993, p. 73-82.

[14] G. Battistoni et al, Resistive cathode transparency. Nucl. Instr. Methods 202 (1982) 459.

[15] G. Bencze et al, Position and Timing Resolution of Interpolating Cathode Strip Chambers in the Test Beam. preprint BNL-CSC-94-2, technical notes: CMS TN/94243 and RD5 TN/94-03, 1994). 


\section{FIGURE CAPTIONS}

Fig. 1: Absolute gain for over-coated chambers with different surface resistivity. The gain was measured for $6 \mathrm{keV}$ X-rays at $10^{3} \mathrm{~Hz} / \mathrm{mm}^{2}$ in Ar-DME (50:50).

Fig. 2: Dark current and surface resistivity of diamond over-coated MSGC\#2 as a function of anode-cathode voltage.

Fig. 3: Measurement of the long term evolution of the MSGC\#2 pulse height in the high (ph1) and low (ph2) irradiated areas, and in the single-wire counter (SW), (uncorrected data). Radiation rate of $2^{*} 10^{5} \mathrm{~Hz} \mathrm{~mm}^{-2}$ at the charge gain $\mathrm{M}=1500$.

Fig. 4: Normalized gain and energy resolution as a function of the integrated avalanche charge for two DLC over-coated MSGCs on D-263 glass with grounded back-plane electrode.

Fig. 5: Gain along the group of strips in the heavily irradiated region of MSGC\#2, damaged during intensive operation. The solid line represents the pulse height and the dashed line corresponds to the energy resolution. The width of the region of lower pulse height and worsened energy resolution corresponds to the width of the irradiated area.

Fig. 6: Initial pulse height slewing as a function of integrated charge for various avalanche current densities. Different spots of $5 \mathrm{~mm}^{2}$ each were been irradiated in MSGC\#2. The charge gain was set to $M=1500$ at the beginning of each measurement.

Fig. 7: Measurement of gain recovery in MSGC\#2. The detector has been exposed to an avalanche current of $7 \mathrm{nA} \mathrm{mm}{ }^{-2}$ for periods of about 12 hours. Between the operation periods the chamber was switched off with radiation source removed.

Fig. 8: Rate capability of the DLC over-coated MSGCs with different surface resistivity. The absolute value of charge gain at $10^{3} \mathrm{~Hz} / \mathrm{mm}^{2}$ was 1500 in each measurement. The over-coated MSGC with surface resistivity of $2.4^{*} 10^{15} \Omega / \square$ exhibits good stability of the gain up to $2 * 10^{6} \mathrm{~Hz} / \mathrm{mm}^{2}$.

Fig. 9: Normalized gain and energy resolution as a function of the integrated avalanche charge for the DLC over-coated MSGC on D-263 glass with chromium strips. Surface resistivity $2.2^{*} 10^{16} \Omega / \square$. The strength of the drift field was $470 \mathrm{~V} / \mathrm{mm}$ during the measurement.

Fig. 10: Measurement of the radiation hardness of the DLC layer on the MSGC with chromium strips on D-263 glass. Relative gain stability during the high dose irradiation of the unpowered detector.

Fig. 11: Resistivity of a thin DLC layer as a function of the temperature of baking in nitrogen atmosphere. DLC deposition was made by ICMC on samples \#1 and \#2. Sample \#3 was prepared by SURMET Co. 


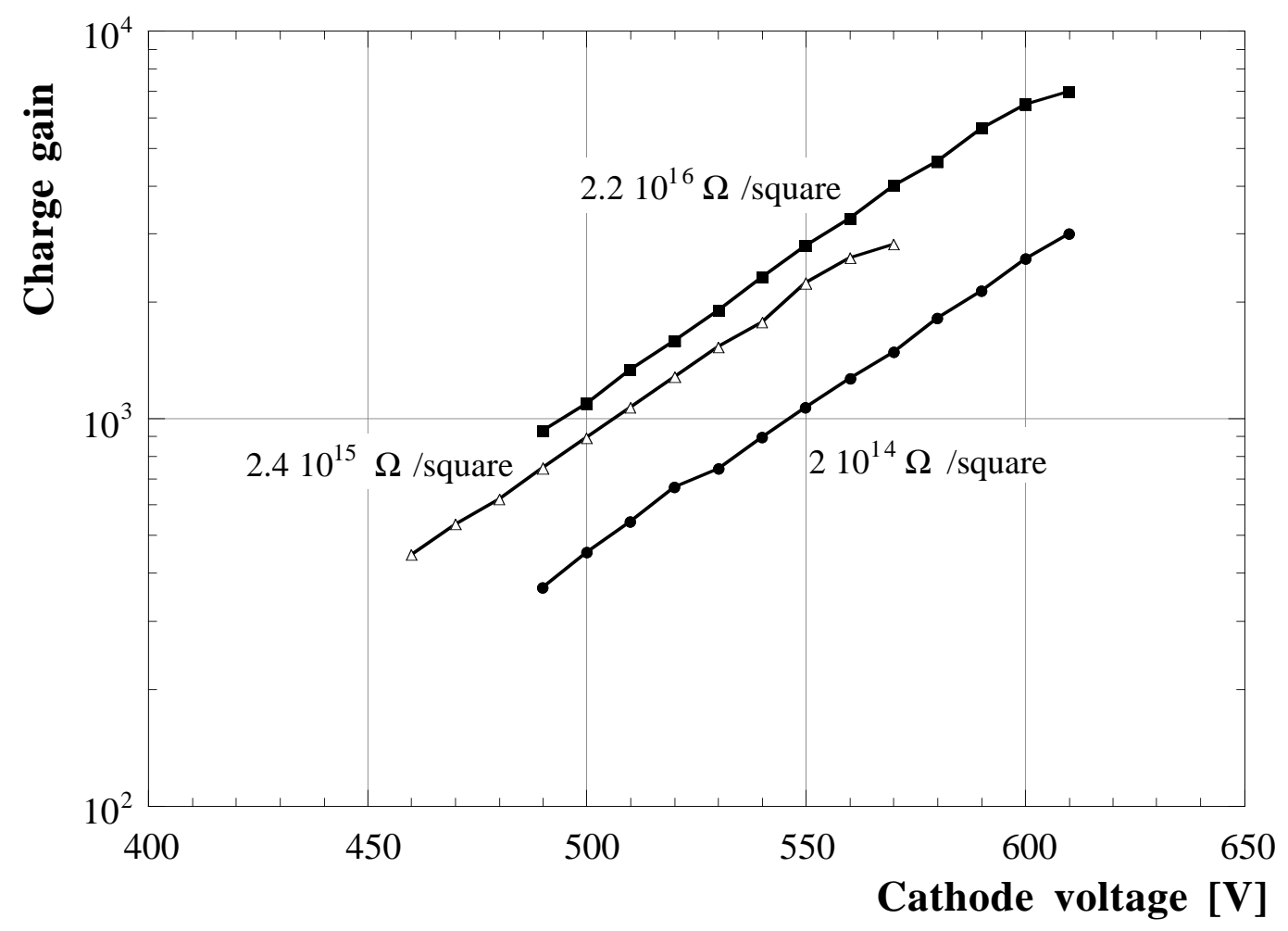

Fig. 1

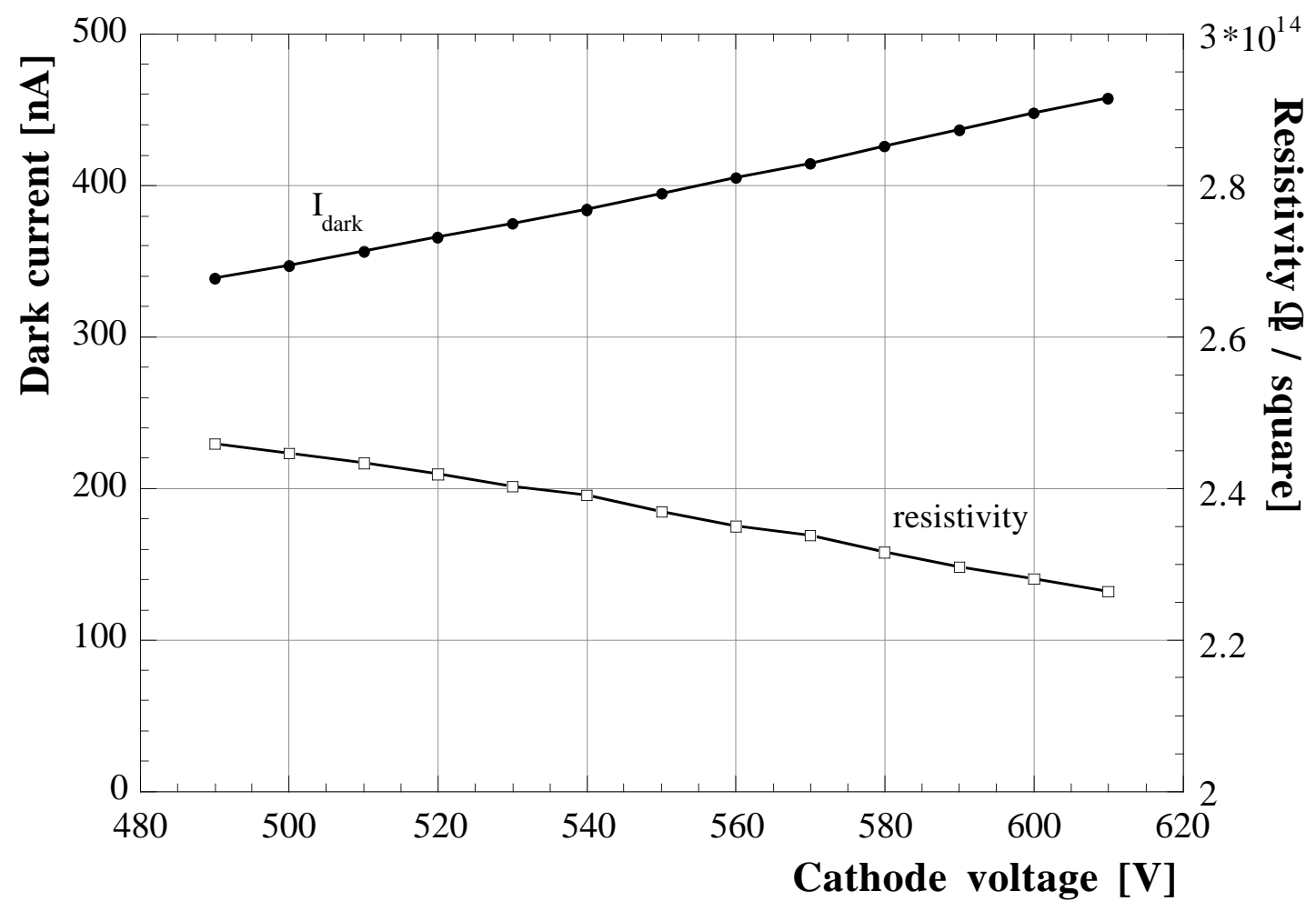

Fig. 2 


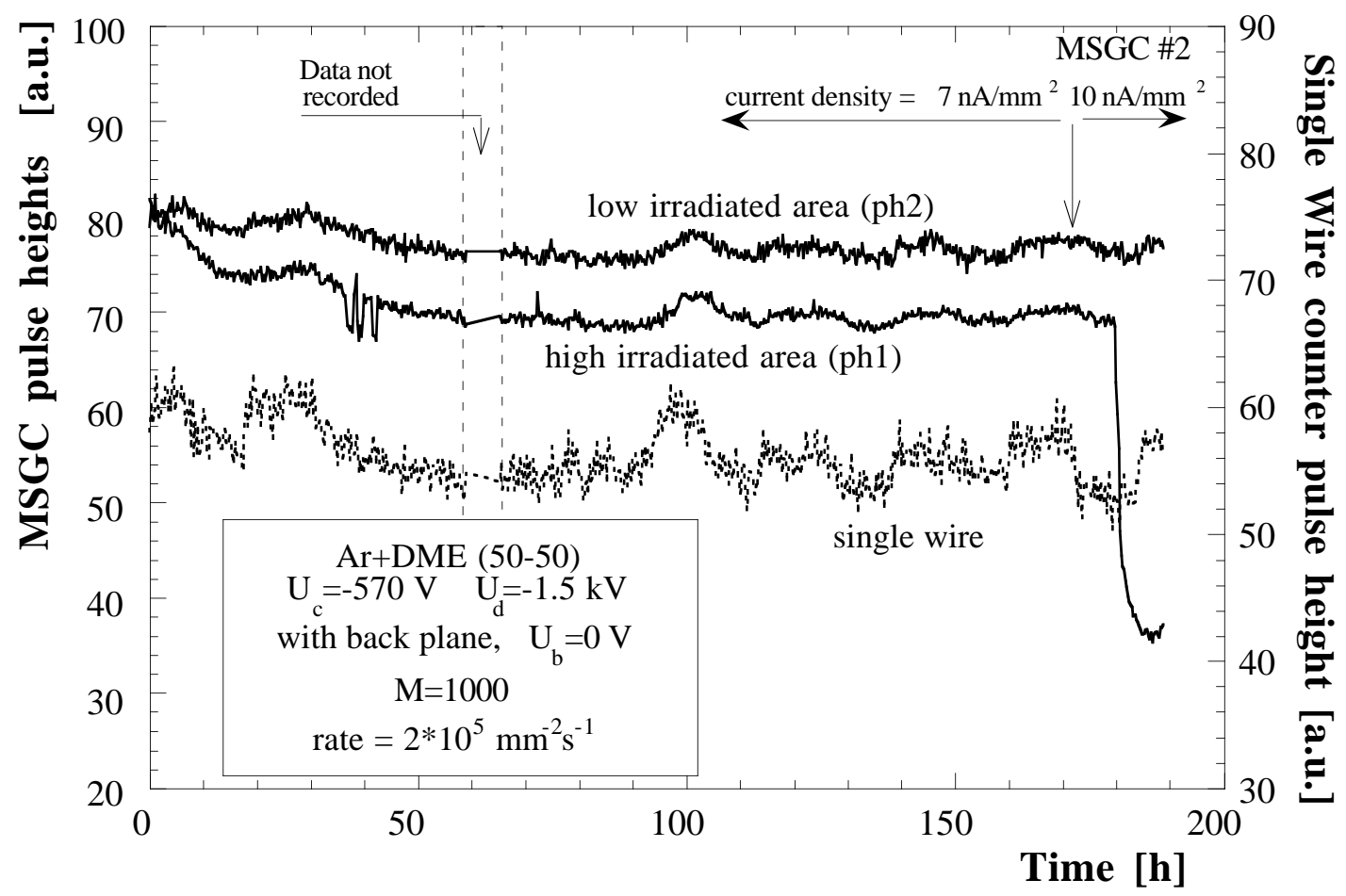

Fig. 3

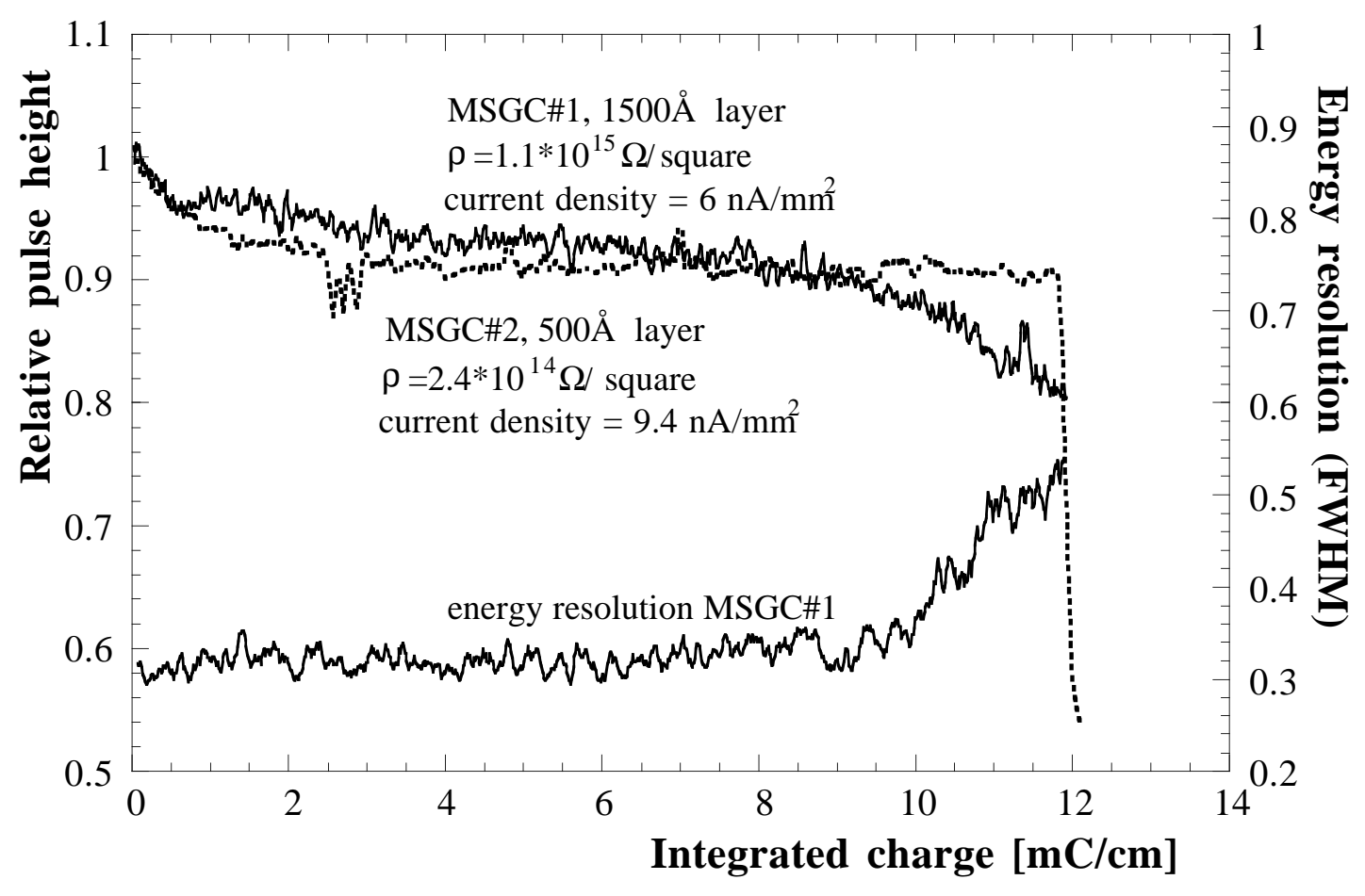

Fig. 4 


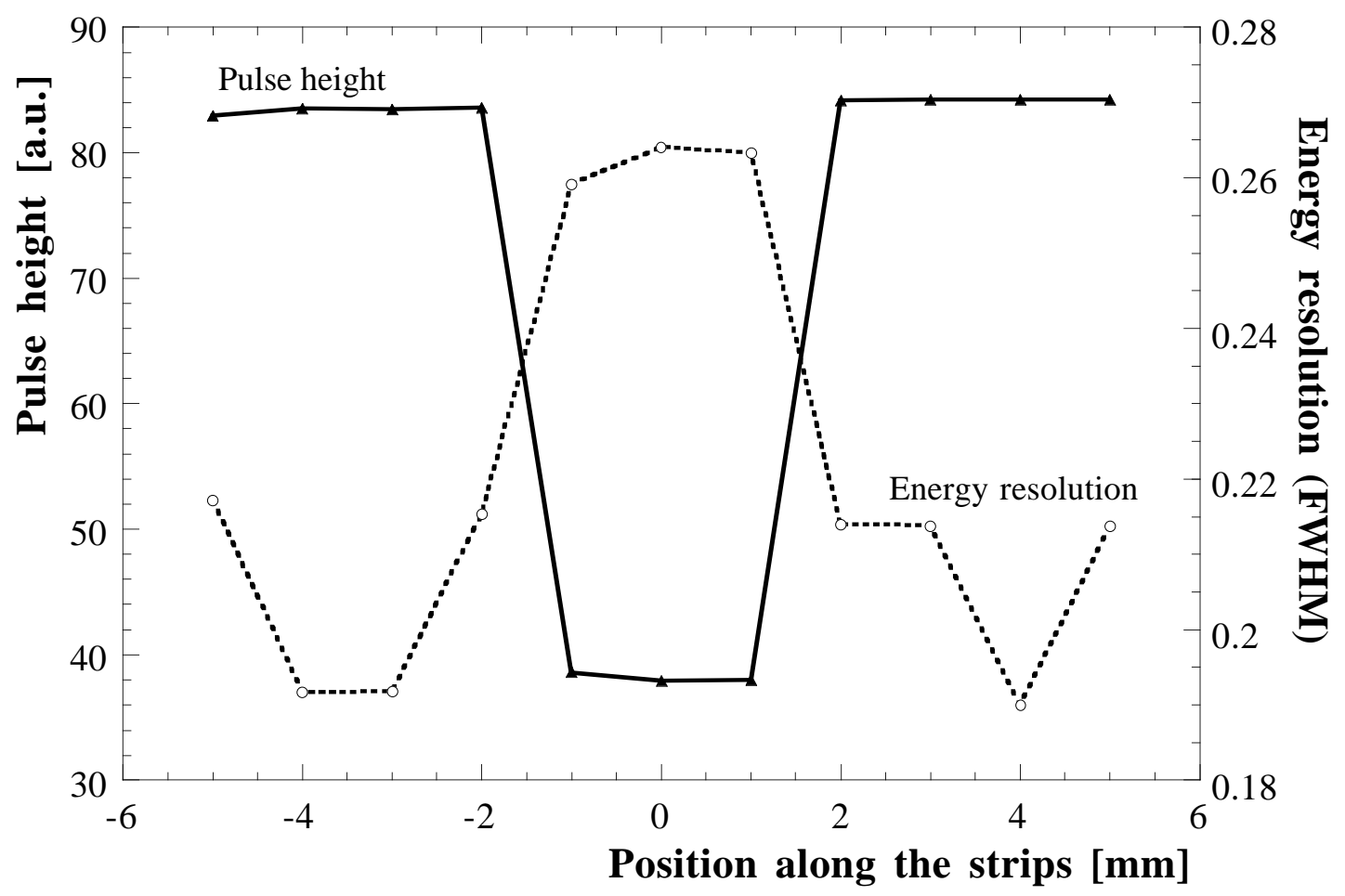

Fig. 5

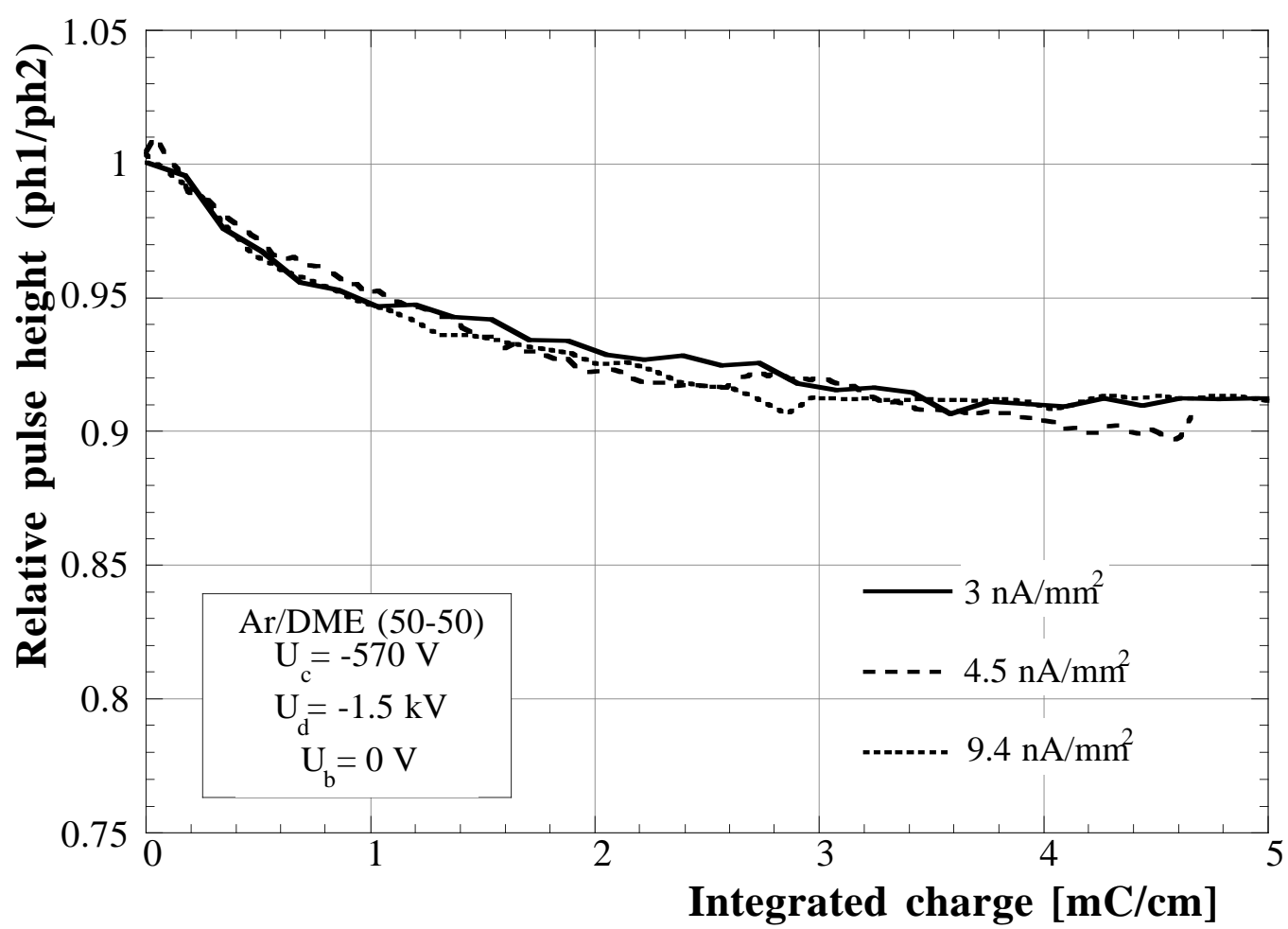

Fig. 6 


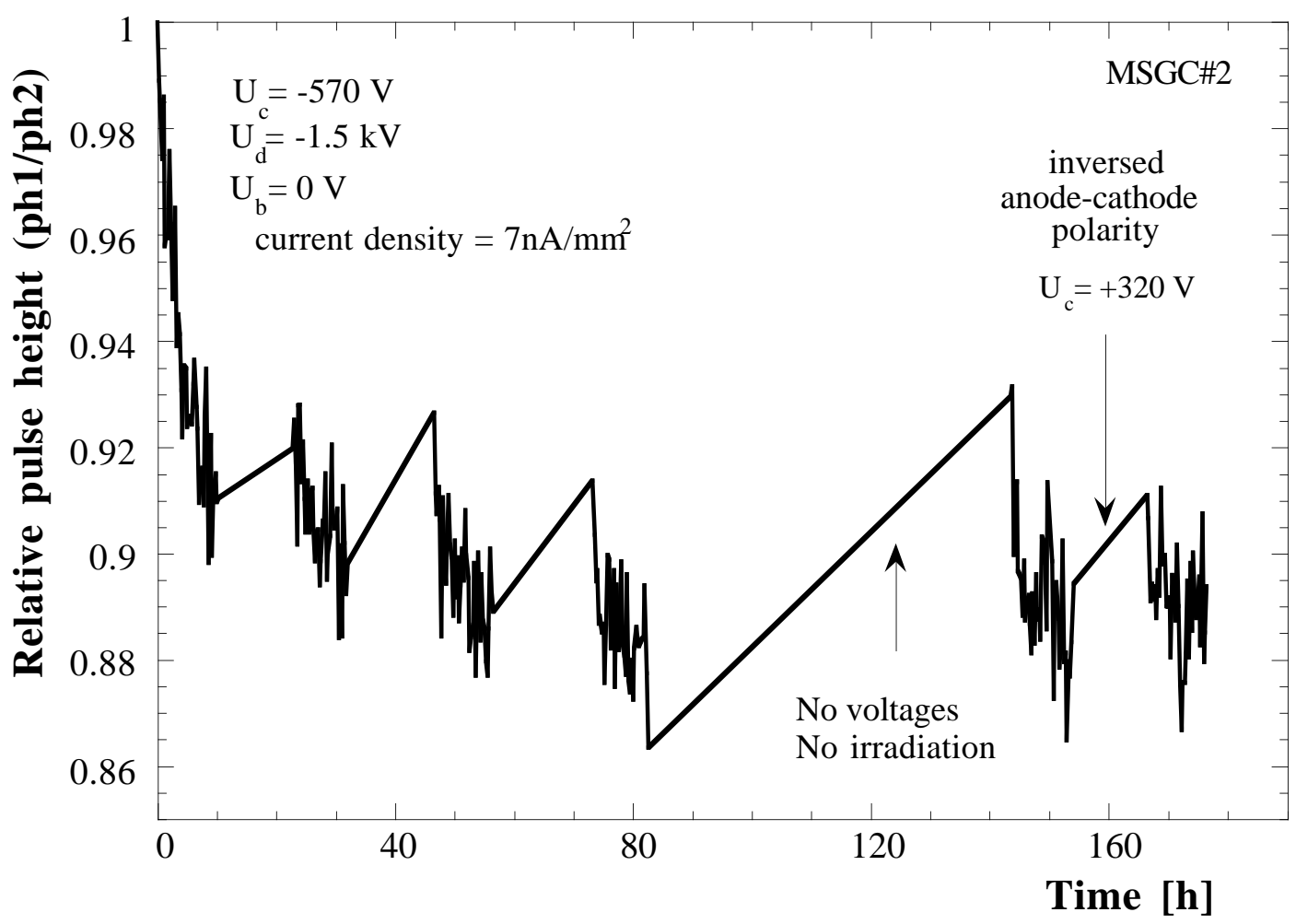

Fig. 7

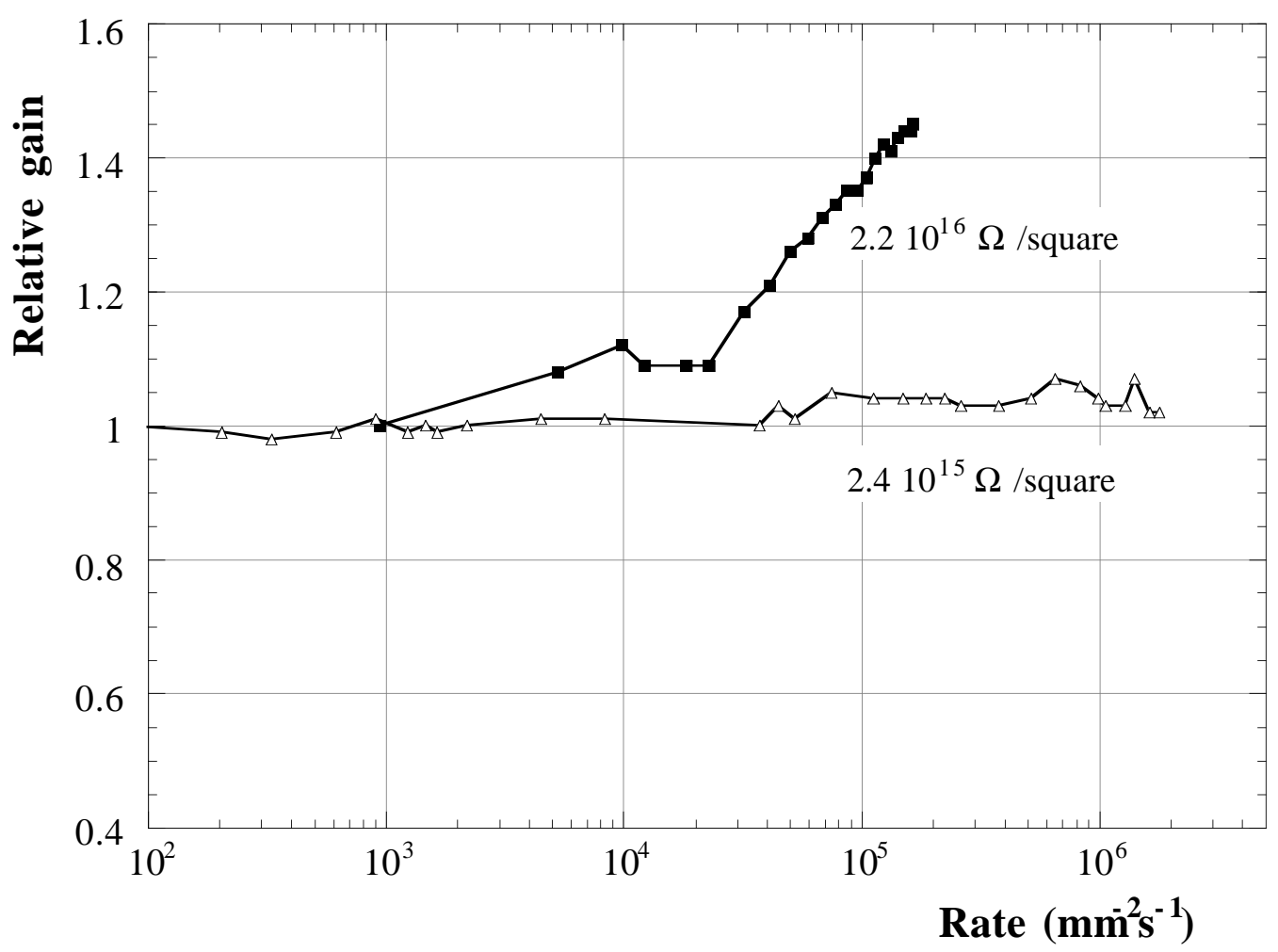

Fig. 8 


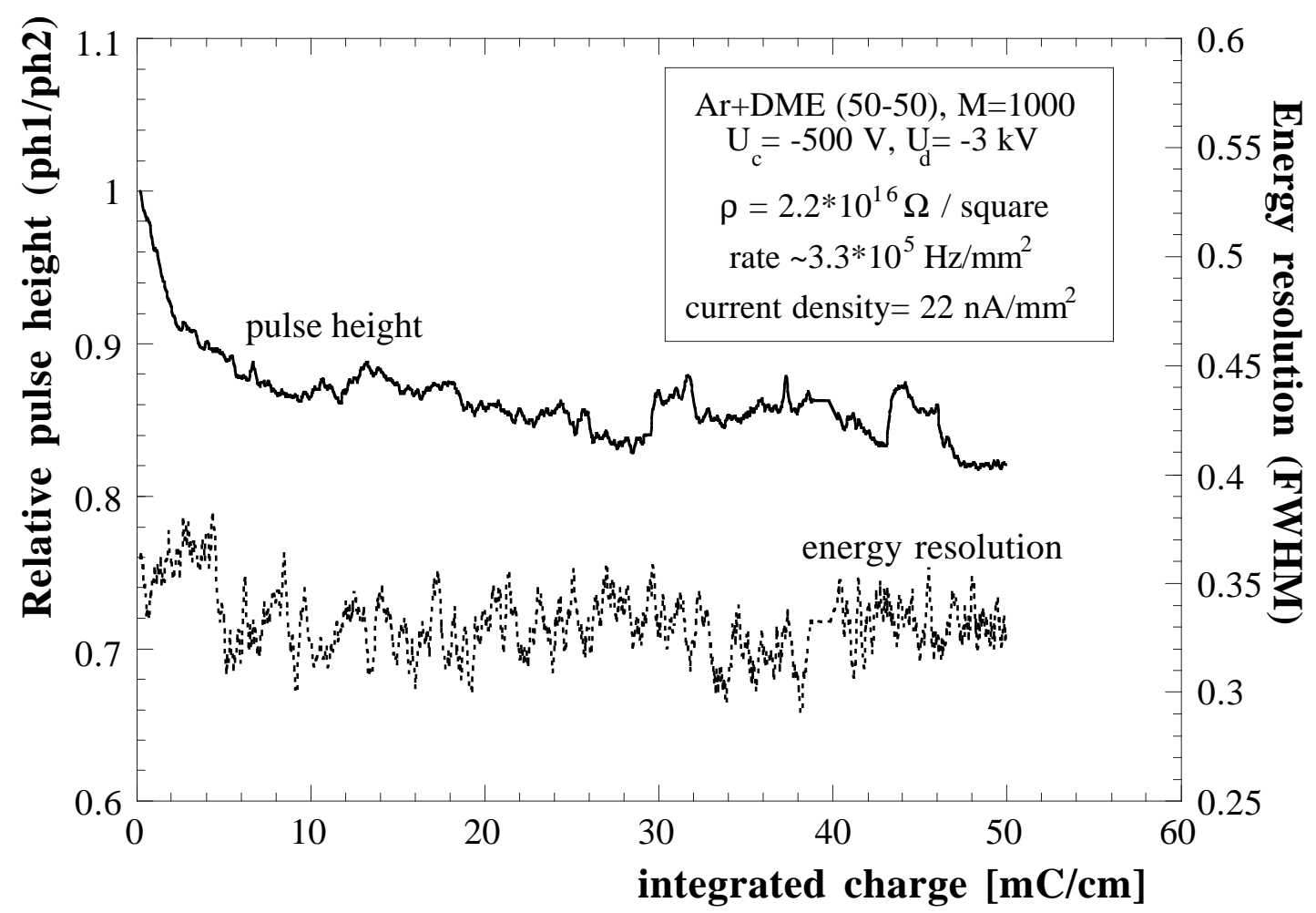

Fig. 9

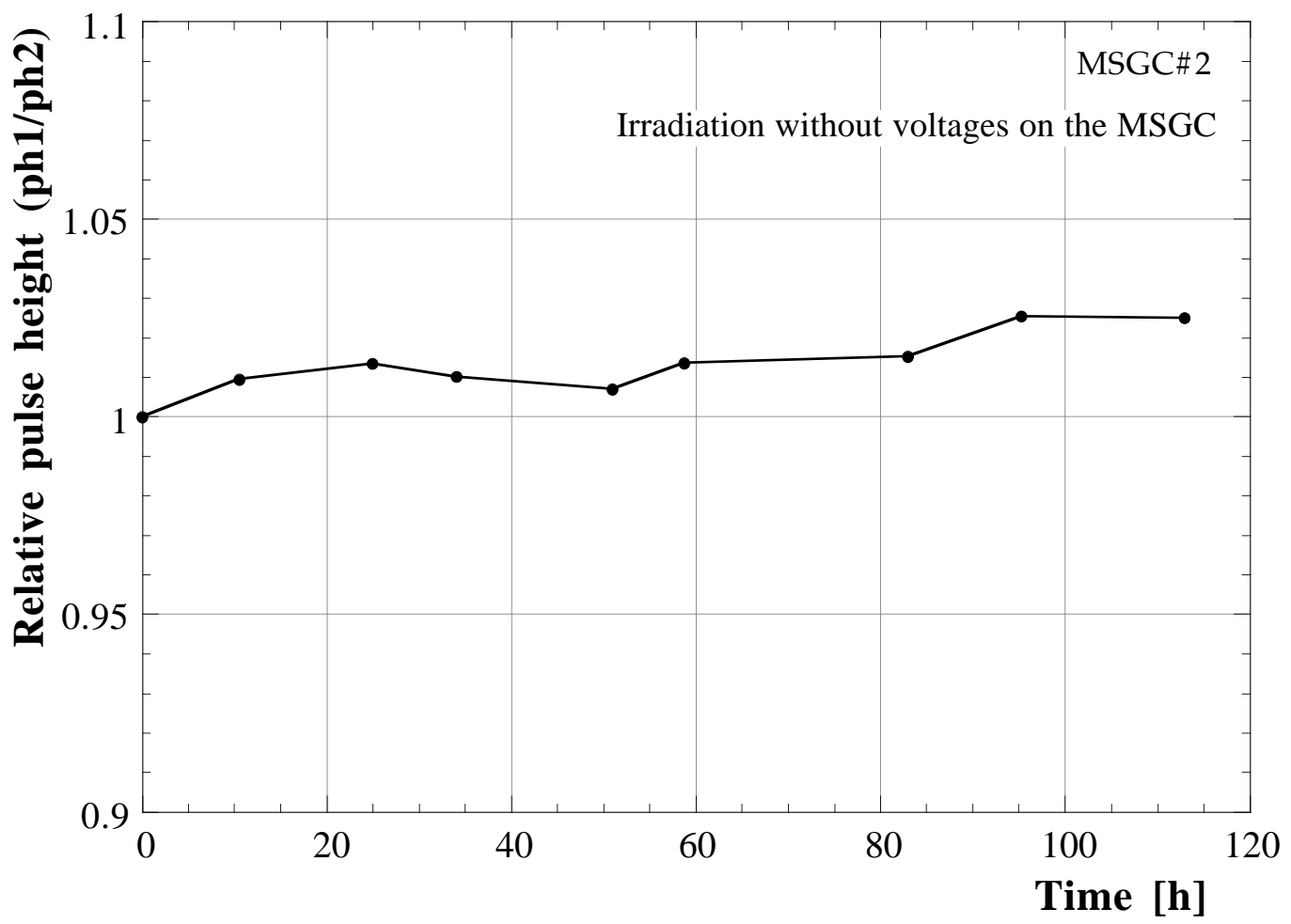

Fig. 10 


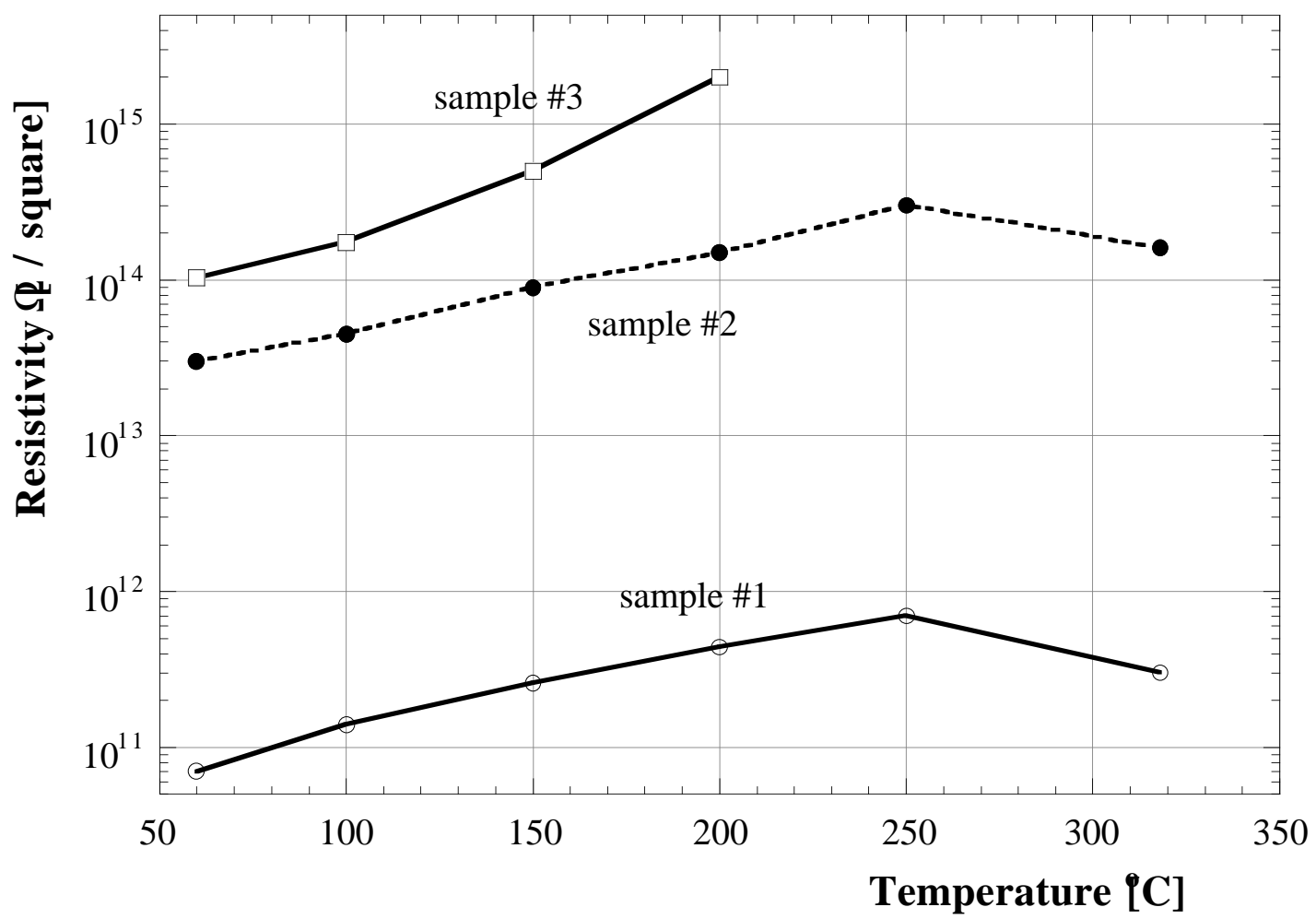

Fig. 11 